\title{
DESIGUALDAD DE GÉNERO EN LA INVESTIGACIÓN MÉDICA ESPAÑOLA: UN CASO PARADIGMÁTICO
}

\author{
(D) J. Eliseo Valle Aparicio \\ (D) Ángel San Martín AlonsoII \\ (D) Raquel Valle Escolano III \\ I Universidad de Valencia (UV), Valencia, España; jevalle@uv.es \\ II Universidad de Valencia (UV), Valencia, España; angel.sanmartin@uv.es \\ III Universidad Carlos III de Madrid (UC3M), Madrid, España; valle.escolano@gmail.com
}

\section{Resumen}

Con el objeto de evaluar la desigualdad de género en la investigación científica médica, se analizan las bases de datos institucionales de investigación de la Universidad de Valencia (2000-2018), de las facultades de ciencias de la salud (Medicina y Odontología, Farmacia, Enfermería y Fisioterapia), considerando sexo, categoría académica, edad y especialidad clínica de los investigadores/as. Los académicos son responsables de un número abrumador de proyectos y contratos de investigación, de importes mucho más elevados que los dirigidos por sus colegas mujeres. El estudio revela también segregación horizontal y una brecha salarial de género significativa, subrayando la necesidad de políticas que optimicen el talento y el trabajo de las científicas médicas españolas en beneficio de la sociedad.

INVESTIGACIÓN • DESIGUALDAD DE GÉNERO • CIENCIAS MÉDICAS • UNIVERSIDAD

\section{DESIGUALDADE DE GÊNERO NA PESQUISA MÉDICA ESPANHOLA: UM CASO PARADIGMÁTICO}

\section{Resumo}

Com o objetivo de avaliar a desigualdade de gênero na pesquisa científica médica, foram analisadas as bases de dados institucionais de um estudo da Universidade de Valencia (2000-2018) das faculdades de ciências da saúde (Medicina e Odontologia, Farmácia, Enfermagem e Fisioterapia) considerando sexo, categoria acadêmica, idade e espacialidade clínica dos/as pesquisadores/as. Os acadêmicos são responsáveis por um significativo número de projetos e contratos de pesquisa, com valores muito mais elevados que os dirigidos por suas colegas mulheres. O estudo também revela segregação horizontal e uma notável brecha salarial de gênero, destacando a necessidade de políticas que otimizem o talento e o trabalho das cientistas médicas espanholas em benefício da sociedade.

PESQUISA • DESIGUALDADE DE GÊNERO • CIÊNCIAS MÉDICAS • UNIVERSIDADE 


\section{GENDER INEQUALITY IN SPANISH MEDICAL RESEARCH: A PARADIGMATIC CASE}

\section{Abstract}

In order to assess gender inequality in medical scientific research, the institutional research databases of the University of Valencia (2000-2018), of the schools of health sciences (Medicine and Dentistry, Pharmacy, Nursing and Physiotherapy) are analyzed, considering sex, academic category, age and clinical specialty of the researchers. Male academics are responsible for an overwhelming number of research projects and contracts, also of a much higher amount, than those led by their female colleagues. The study also reveals horizontal segregation and a significant gender pay gap, highlighting the need for policies that optimize the talent and work of Spanish medical women scientists for the benefit of society.

RESEARCH・GENDER INEQUALITY • MEDICAL SCIENCES • UNIVERSITY

\section{INÉGALITÉ DE GENRE DANS LA RECHERCHE MÉDICALE ESPAGNOLE : UN CAS PARADIGMATIQUE}

\section{Résumé}

Pour mesurer les inégalités de genre dans le domaine de la recherche médicale, ce travail a analysé les bases de données rélatives aux recherches de l'Université de Valence (2000-2018) provenant des facultés des sciences de la santé (Médecine, Dentaire, Pharmacie, Soins Infirmiers et Kinésithérapie), en prenant en compte le sexe, la catégorie universitaire, l'âge et la spécialité clinique des chercheur/ chercheuses. Les hommes sont responsables d'un grand nombre de projets et de contrats de recherche dont les valeurs sont beaucoup plus élevées que pour ceux menés par leurs collègues femmes. En outre, l'étude a montré qu'il existe une ségrégation horizontale ainsi qu'un fort écart salarial entre les sexes, ce qui renforce l'idée que sont nécessaires des politiques qui optimisent les talents et le travail des scientifiques espagnoles du médical, pour le bénéfice de la société dans son ensemble.

RECHERCHE • INÉGALITÉ DES SEXES • SCIENCES MÉDICALES • UNIVERSITÉ 

a las Facultades de Medicina y en general de ciencias de la salud en la universidad española se ha ido incrementando de forma extraordinaria, de modo que en la Universidad de Valencia éstas representan el 70,3\% de los estudiantes en las titulaciones de grado, y el 74,1\% en las enseñanzas de master. En los estudios de doctorado descienden muy ligeramente, siendo no obstante todavía una importante mayoría del alumnado (63,2\%). Dichos datos revelan, sin duda, pasos importantes para lograr la igualdad de género. Sin embargo, con ser cruciales, todavía no han dejado sentir sus efectos, consiguiendo revertir determinadas tendencias de inequidad que todavía están muy presentes en la universidad española. La primera de ellas, la constatación de que el referido predominio de estudiantes mujeres en ciencias de la salud no se corresponde con la distribución por sexos de las personas que eligen realizar una carrera académica en la universidad como personal docente e investigador, donde las mujeres ya comienzan siendo minoría, frente a una proporción importante de varones, que continúa en ascenso a medida que se avanza hacia figuras de mayor rango y categoría.

Junto a este abandono femenino, explicativo de una más débil presencia en todos los colectivos de personal docente e investigador, y singularmente en las categorías académicas superiores -el denominado embudo académico- (Paulus et al., 2016), diversos estudios han constatado la fuerza de otras tendencias que corren parejas a la anterior, como las diferencias de liderazgo entre hombres y mujeres (Monroe et al., 2015; Rochon et al., 2016; Pingleton et al., 2016), y la desigualdad de género en la consecución de fondos para la investigación (Bates et al., 2016; Ministerio de Ciencia, Innovación y Universidades [MCI], 2018a). Todos estos factores están en la base de la infrarepresentación de las mujeres y su perpetuación, especialmente en los altos cargos académicos (Carr et al., 2018), incluso en sociedades modernas con políticas y planes a favor de la igualdad de género (Plank-Bazinet et al., 2016).

$\mathrm{Al}$ abordar el tema de la investigación académica en particular, nuevamente encontramos diferencias de productividad y desempeño entre hombres y mujeres (Besselaar \& Sandstrom, 2016; Lee \& Ellemers, 2015), que dejan sentir sus efectos en la producción científica. Los investigadores varones obtienen más fondos de investigación (Waisbren et al., 2008; Ceci \& Williams, 2011; Lee et al., 2015; Ovseiko et al., 2016; MCI, 2018 a), publican más artículos (Astegiano et al., 2019; Raj et al., 2016), obtienen un número más elevado de citas por sus trabajos y aportaciones científicas (Alloza Frutos et al., 2012; Raj et al., 2016; European Commission [EC], 2019), dirigen y ocupan puestos de liderazgo en revistas y comités científicos en mayor medida (Mauleón et al., 2013; EC, 2019), reciben más y mejores premios y reconocimientos científicos, obtienen más patentes (EC, 2019) y demuestran mayor habilidad para formar parte de redes de investigación, desarrollando así esquemas de colaboración internacional y de coautoría más valiosos que sus colegas mujeres (European Institute for Gender Equality [EIGE], 2019b; Shannon et al., 2019).

Atribuir tales hechos a explicaciones meritocráticas como único elemento justificativo no parece muy convincente, y de ahí que la literatura académica ofrezca al respecto una amplia gama de factores ampliamente aceptados, algunos personales y actitudinales, et al. estructurales, sociales e institucionales (Fox et al., 2011; EIGE, 2016), que pueden explicar las diferencias de género en la productividad investigadora.

Entre los primeros, con efectos perjudiciales en las carreras profesionales de las mujeres, destacan la maternidad y en general una mayor dedicación a temas domésticos y familiares (Fox et al., 2011; EIGE, 
2016; EC, 2019; Bowen et al., 2018; Shannon et al., 2019), con el consiguiente impacto en su trayectoria investigadora, en un contexto de escasas dotaciones para la investigación y gran competitividad. También se traen a colación las propias percepciones femeninas, en cuanto que las investigadoras no avanzan con idéntica confianza en su carrera profesional (Butkus et al., 2018), poseen estilos de liderazgo e interacción diferentes a los de los hombres (Carr et al., 2018), así como una menor ambición profesional, una vez que han alcanzado la estabilidad en la universidad (Escolano, 2006), lo que explicaría asimismo sus menores demandas al negociar (Buckley et al., 2000), un factor importante en los contratos de investigación. También se advierte una mayor planificación estratégica por parte de los académicos varones, que suelen focalizar sus trabajos en temáticas y enfoques más orientados a lo que es tendencia, frente a las investigadoras, más ajenas a tales cálculos. La planificación de la carrera académica con un sentido más pragmático y rentable se plasma de modo notable en la faceta investigadora, esencial para avanzar en la trayectoria profesional del personal académico (Escolano, 2006).

Las académicas que forman parte de los grupos de investigación consolidados, tampoco suelen como regla general liderarlos, sino que en muchos casos ocupan una posición secundaria, aunque en muchos casos tengan una mayor carga de trabajo. Los investigadores responsables que lideran tales equipos resultan acreedores del prestigio que supone conseguir fondos o alcanzar resultados relevantes, en mucha mayor medida que sus colegas mujeres, que además en muchos otros casos desarrollan elecciones y trayectorias profesionales no tan focalizadas en la investigación, y mucho más centradas en la enseñanza (Wenneras \& Wold, 1997; EC, 2019).

Desde la perspectiva social e institucional, diversos estudios han destacado la vigencia de sesgos de género, aun inconscientes, y de estereotipos que favorecen a los hombres. Se señala que quienes valoran las solicitudes de fondos para proyectos de investigación y los artículos candidatos a ser publicados, así como las personas que acceden a los mismos cuando se difunden, tienden a sobrevalorar en términos generales los trabajos de autoría masculina, considerando en menor medida a aquellos realizados por académicas, ya sea por la temática, el enfoque, la metodología, etc. (Shen, 2013; Leslie, 2015; Pingleton et al., 2016; Besselaar et al., 2016; Pattani et al., 2018). Ello se hace en ocasiones de forma no del todo consciente, ya que los estereotipos sobre hombres y mujeres siguen estando plenamente vigentes, y la excelencia investigadora tiende a relacionarse más implícitamente con imágenes masculinas, dada la falta histórica de referentes femeninos en la ciencia y la ligazón de la imagen de las mujeres con ámbitos privados, reñidos con las exigencias de tiempo y esfuerzo que la investigación científica lleva consigo (Alloza Frutos et al., 2012; Pattani et al., 2018). La tradición intelectual masculina sigue siendo predominante en el ámbito de las ciencias médicas. Todos los factores anteriores conforman un ambiente académico hostil (Fox et al., 2011), que ofrece a las mujeres menos apoyo, orientación y patrocinio que a los hombres (Yedidia \& Bickel, 2001; Westring et al., 2016), consolidando el techo de cristal en la carrera investigadora (MCI, 2018a).

El funcionamiento de la universidad española, por otro lado, nos remite a la extraordinaria importancia de los contactos, las relaciones, la pertenencia a redes, aun cuando los procedimientos de acceso a los fondos de proyectos de investigación competitivos sean formalmente abiertos y objetivos (EIGE, 2016). Y las mujeres en tales ámbitos informales de actuación no están tan presentes y tienen menos contactos. Los académicos varones poseen mayor destreza a la hora de manejarse en grupos y lobbies académicos, lo que es clave en el terreno de la investigación, por la influencia de las redes de colegas conocidos, así como por la conveniencia de trabajar en grupos, en muchos casos interdisciplinares. Y en el caso de los contratos de transferencia de investigación, las relaciones son prácticamente el elemento fundamental a la hora de conseguirlos, presuponiendo la calidad investigadora de ambos sexos. Las académicas no se manejan tan bien en tales redes, ni dedican el esfuerzo necesario para participar en las mismas, ni son invitadas a hacerlo. Y en este terreno es donde aparece otro elemento importante, la brecha salarial (Connolly \& Holdcroft, 2009; Shen, 2013; Bates et al., 2016; EC, 2019; EIGE, 2019b), 
que surge de las diferencias retributivas entre hombres y mujeres en las investigaciones de ciencias médicas, dado que, con cargo a los fondos de dichos contratos, el personal docente e investigador de las universidades españolas percibe importantes retribuciones complementarias. Si bien los salarios ordinarios son iguales para hombres y mujeres, al encontrarnos en una universidad pública, por esta vía surge una brecha salarial que beneficia de forma importante a los académicos varones.

Existe una fuerte interacción entre los dos tipos de elementos apuntados (Zuckerman, 2001), porque los factores sociales e institucionales tienden a reforzar los personales, como las elecciones y los pasos que las mujeres llevan a cabo en sus carreras profesionales. Al final, todas estas selecciones personales y sociales a lo largo del tiempo conducen a desventajas acumulativas para las mujeres académicas (Eurostat, 2019), con carreras de investigación más lentas y mucho menos relevantes (MCI, 2018b).

En los últimos tiempos, la promoción de la igualdad de género en la investigación y la innovación es un compromiso asumido a nivel europeo, tal como estableció el Programa Horizonte 2020, que busca garantizar la promoción eficaz de la igualdad entre hombres y mujeres mediante el equilibrio de género en los equipos de investigación, tanto en la toma de decisiones (paneles, comités de expertos, asesores..), como en la integración de la dimensión de género en el contenido de la investigación y la innovación (formación, enfoques, financiación específica..), reforzando así el compromiso de equipos e instituciones participantes. En España se han puesto en marcha importantes medidas de discriminación positiva, establecidas por la ley de igualdad estatal y las normativas autonómicas, destacando la creación en 2005 de la Unidad de Mujeres y Ciencia como organismo responsable de la incorporación de la perspectiva de género en ciencia, tecnología e innovación en nuestro país. También las universidades españolas han integrado acciones diversas en sus planes de igualdad (en particular, en la Universidad de Valencia está actualmente vigente su tercer Plan de Igualdad), muchas de las cuales se centran en lograr una mayor equidad en la investigación, así como en analizar el avance de las académicas en sus carreras profesionales.

Este estudio evalúa la participación y el papel de mujeres y hombres en la investigación académica en el campo de las ciencias de la salud en la Universidad de Valencia (UV), España, una institución con más de cinco siglos de existencia, que destaca por sus antecedentes y su excelencia académica y de investigación, especialmente relevante en ciencias médicas. Sus características la convierten en una institución paradigmática de las universidades públicas españolas, donde los datos de categorías académicas, participación, protagonismo y liderazgo de hombres y mujeres son similares, lo que sugiere que las tendencias observadas en este estudio pueden tener una generalidad más amplia.

La presente investigación busca conocer la participación y el protagonismo de mujeres y hombres en la investigación académica en el ámbito de ciencias de la salud y determinar si existe una situación igualitaria en el acceso a los fondos y en la dirección y responsabilidad de los proyectos y actividades investigadoras, incidiendo en cuáles son los factores que explicarían eventuales desigualdades de género en una sociedad que ha experimentado profundas transformaciones, y con políticas de igualdad de género en marcha. También se busca averiguar si existe una brecha salarial entre los académicos de ambos sexos, por vía de las eventuales retribuciones que pueden percibir los investigadores/as con cargo a los contratos de investigación mediante los cuales se transfieren los resultados de investigación a la sociedad.

\section{Método}

Los fondos destinados a la investigación en las universidades españolas se logran fundamentalmente a través de dos vías. La primera es la participación de los profesores/as e investigadore/as y sus grupos de investigación en convocatorias competitivas, mediante las que las Administraciones públicas (el Estado, las Comunidades Autónomas o la Unión Europea) subvencionan de forma competitiva proyectos de investigación. El segundo origen de los recursos destinados a la investigación académica, lo constituye la firma de contratos y convenios del personal docente e investigador con empresas e instituciones 
públicas y privadas, previstos en el artículo 83 de la Ley Orgánica 6/2001 de Universidades (LOU), mediante los cuales se lleva a cabo desde la universidad la transferencia a la sociedad de los resultados de la investigación científica y técnica, realizada por encargo de sus destinatarios.

Con el objeto de analizar desde una perspectiva de género los recursos dedicados a la investigación en el área de ciencias de la salud, el estudio utiliza como fuentes los datos institucionales anonimizados de las bases de datos de investigación de la Universidad de Valencia, que el equipo del gobierno académico proporcionó específicamente para la realización de este estudio, previa solicitud.

Se han utilizado tres bases de datos institucionales, explotando todas las variables de interés al objeto de nuestra investigación:

1. Base de datos de fondos de investigación: Esta base comprende la totalidad de los proyectos de investigación, así como de los contratos de I + D + i suscritos en el periodo objeto de análisis, dirigidos por profesores e investigadores de ciencias de la salud, hombres y mujeres, como investigadores principales.

2. Base de datos de nóminas, módulo correspondiente a pagos a investigadores, derivados de su participación en contratos del artículo 83 de la LOU.

3. Base de datos de Grupos de investigación inscritos en el Registro de Estructuras de Investigación de la Universitat de València (REIUV). Se han explotado los datos de los grupos de Ciencias de la Salud, disponibles para la anualidad 2018, última de nuestro análisis.

1) Los datos proporcionados abarcan prácticamente dos décadas (2000-2018) y comprenden la información completa relativa a todos los recursos dedicados a la investigación vigentes en dicho lapso temporal, procedentes ya de ayudas públicas que financian proyectos de investigación, desarrollo e innovación (en adelante, $\mathrm{I}+\mathrm{D}+\mathrm{i}$ ) -en el marco del Plan Estatal, proyectos europeos o subvenciones a la investigación del gobierno valenciano-, así como los generados por contratos de transferencia de resultados de investigación suscritos y en vigor en tal periodo.

Los académicos, hombres y mujeres responsables de los mismos como investigadores principales, pertenecen a la totalidad de los departamentos y disciplinas en el área de las ciencias de la salud. En concreto, son personal docente e investigador adscrito a la Facultad de Medicina y Odontología, a la Facultad de Farmacia, a la Facultad de Enfermería y a la Facultad de Fisioterapia.

Se han explotado y analizado datos para evaluar la distribución por género de los fondos de investigación para todo el período de estudio (2000-2018), no solo global o acumulativamente, sino también longitudinalmente, con el propósito de detectar los eventuales cambios a lo largo del tiempo y verificar las tendencias observadas en su evolución. Para esta última finalidad se han establecido varios intervalos, dentro del lapso temporal global, en concreto los periodos 2000-2005: 2006-2010; 20112015; y 2016-2018.

Por otro lado, se han filtrado los datos considerando tres variables además del sexo de los investigadores, para descubrir en qué medida cada una de ellas puede tener incidencia, influyendo y modulando los resultados generales.

La primera es la categoría académica, ya que el rango profesional suele ser decisivo para obtener fondos para la investigación, tal y como han puesto de manifiesto numerosos estudios (por todos, Waisbren et al., 2008). Por ello, se contemplaron adicionalmente todas las cuestiones analizadas tomando en consideración la categoría académica de los investigadores/as, obteniendo resultados diferentes cuatro grupos de profesorado: en primer lugar, para los profesores contratados temporales (un conjunto que engloba al profesorado asociado, así como a los ayudantes doctores, y a alguna otra figura residual); en segundo lugar, se consideró a los miembros del personal docente e investigador que tienen una vinculación permanente con la universidad (profesorado titular de universidad, cated ráticos de escuela universitaria y contratados doctores); los catedráticos/as de universidad, teniendo en cuenta que constituyen la máxima categoría universitaria, se analizaron asimismo de forma monográfica; y de 
idéntico modo se procedió con el profesorado emérito, en cuanto que suelen ser en su práctica totalidad catedráticos/as de universidad, ya que la LOU los cataloga como profesorado que ha prestado servicios destacados a la universidad.

En segundo lugar, utilizamos cuatro rangos de edad para detectar posibles diferencias en el acceso a los fondos de investigación a lo largo de la vida y la carrera profesional de los investigadores e investigadoras universitarios, dado que la edad es un factor esencial en los patrones de dedicación a la carrera profesional, ya por la mayor necesidad de acumular méritos en las primeras etapas, hasta alcanzar una cierta consolidación laboral, ya por la incidencia de las diversas obligaciones familiares y especialmente de tenencia y crianza de hijos/as, especialmente en el caso de las investigadoras. Los rangos empleados han sido cuatro: de 30 a 40 años; de 41 a 50 años; de 51 a 60 años; y finalmente, el grupo de personal docente e investigador mayor de 60 años.

Finalmente, también se han considerado las diferencias de género en la investigación en las diferentes especialidades médicas ( 23 en concreto), analizando los datos que arroja cada una de ellas en cuanto a liderazgo de equipos investigadores y manejo de fondos dedicados a la investigación clínica, tanto en relación a los contratos como a los proyectos de investigación, con el objeto de evaluar si en este ámbito académico nos encontramos, en relación con las cuestiones analizadas, con situaciones que hagan patente la segregación horizontal.

2) Una última cuestión que este estudio aborda para detectar la eventual existencia de una brecha salarial de género, es la relativa a las retribuciones adicionales que perciben los investigadores hombres y mujeres, procedentes de los contratos de investigación en los que participan. Para ello se han analizado las notas de abono tramitadas por investigadores/as de ambos sexos, cuyo montante se ha obtenido explotando la base de datos de nóminas. En particular se han analizado las percepciones correspondientes a los últimos cinco años de datos de nuestro estudio, que cubren el período 2014-2018.

Toda la investigación fue llevada a cabo durante el segundo trimestre de 2019.

\section{Resultados y discusión}

\section{Fondos de investigación}

En primer lugar, si analizamos desde una perspectiva de género el acceso y manejo de fondos dedicados a la investigación médica, de los que los académicos, hombres y mujeres, figuran como investigadores principales para todo el periodo 2000-2018, y comenzando por los proyectos de investigación, resultado de convocatorias competitivas, los varones los controlan mayoritariamente, en mucho mayor medida que sus colegas mujeres, ya que prácticamente doblan las partidas disponibles que poseen para investigar ( $25.681 .957 €$, frente a $13.753 .555 €$, respectivamente). El recuento del número de proyectos de investigación por sexo arroja asimismo un balance favorable a los académicos varones, que lideran 276 proyectos, frente a los 207 en que la responsable es una investigadora mujer. Aunque la diferencia numérica no es excesiva, el hecho de que las cuantías de los fondos de investigación manejados por los hombres prácticamente doblen los controlados por mujeres, nos permite concluir que aquellos consiguen la adjudicación de proyectos con mayor dotación económica que estas últimas. También registramos un predominio masculino en los grupos de investigadores que lideran más de 4 proyectos, de 5 a 9 , o más de 10 proyectos. 
GRÁFICO 1

IMPORTE Y NÚMERO DE CONTRATOS Y PROYECTOS DE INVESTIGACIÓN EN CIENCIAS DE LA SALUD POR SEXO 2000-2018
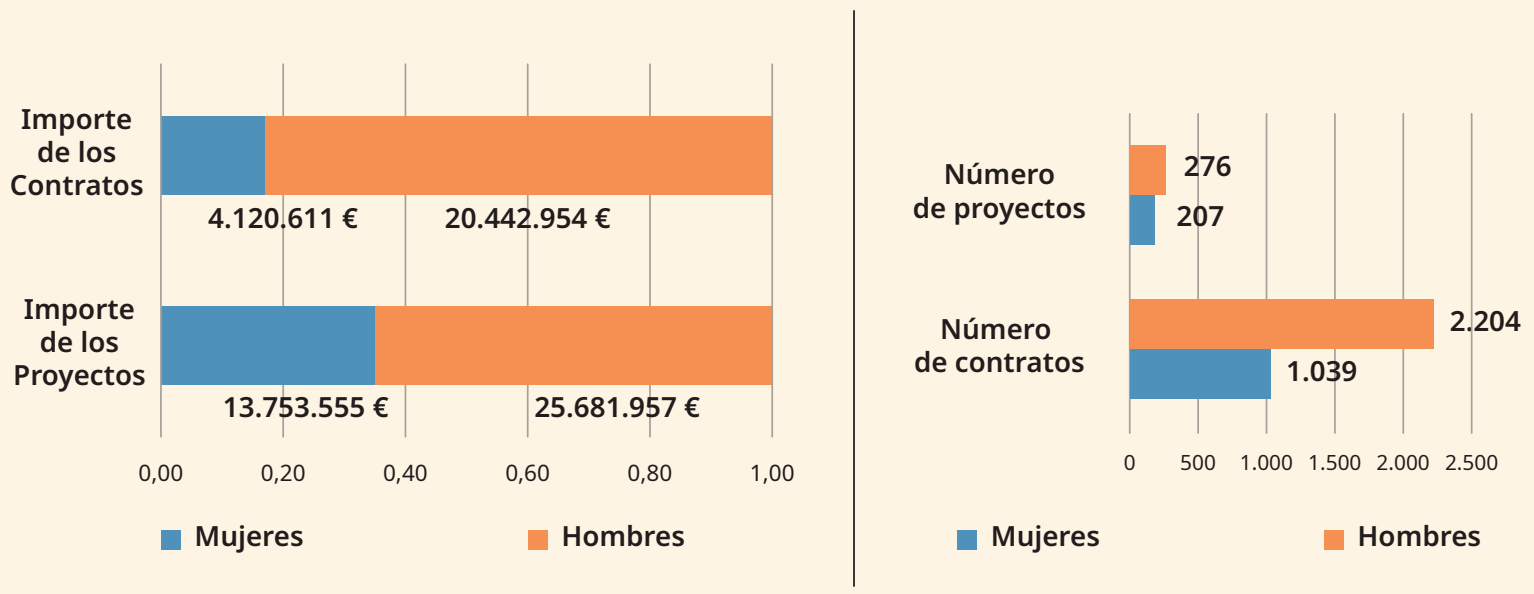

Fuente: Base de datos de investigación de la Universidad de Valencia (2019).

Mucho mayor es el desequilibrio al considerar las cantidades correspondientes a convenios y contratos de investigación, cuya suscripción implica mucho más que ser un investigador/a con solvencia y competencia, ya que supone tener contactos y saber venderse a las instituciones y empresas con las que se firman tales compromisos, que constituyen importantes vías de financiación de la investigación en un país como España, que dedica cantidades modestas a la investigación en los presupuestos públicos. El examen los fondos conseguidos en el tiempo transcurrido desde comienzos del siglo XXI hasta la actualidad permite concluir que los investigadores hombres quintuplican los importes que manejan las académicas que investigan en ciencias médicas $(20.442 .954 €$, frente a 4.120.611 €). Y de nuevo son también ellos quienes aparecen como responsables, en su rol de investigadores principales, de un número mucho mayor de contratos y convenios que sus colegas mujeres (más del doble, 2.204 frente a 1.039 liderados por investigadoras), predominando asimismo claramente como titulares de 3, 4 ó más de 5 de estos convenios y contratos para el periodo analizado. La figura 1 muestra claramente tales brechas de género.

El análisis de la evolución de los importes de contratos y proyectos de investigación por sexo a lo largo del periodo, realizando idéntico estudio para cada uno de intervalos elegidos considerado individualmente (2000-2005: 2006-2010; 2011-2015; 2016-2018), nos permite matizar ligeramente los resultados anteriores. Así, si consideramos los contratos de investigación, si en el primer periodo los investigadores hombres concentran el $90 \%$ de sus importes (de un total de 7.270.779 $€$ ), tales cantidades van en descenso, siendo el 83,2 \% (de 8.662.180 €) en el segundo intervalo, el 75,4\% (de 5.963.662€) en el tercero, para repuntar ligeramente hasta casi el $81 \%$ (de un global de 2.666.945 €) en el periodo 2016-18.

En todo caso, en el estudio global del periodo, se advierte una disminución en los fondos de convenios y contratos para la transferencia de los resultados de la investigación médica de los que son reponsables investigadores hombres, con el correlativo aumento de aquellos que están a cargo de una mujer como investigadora principal, prácticamente de 10 puntos porcentuales.

Distinta ha sido la evolución de los fondos de investigación conseguidos a través de proyectos competitivos, financiados desde la Unión Europea, la Administración estatal o la autonómica. El análisis de los citados intervalos temporales muestra que los varones han visto incrementados los fondos de los que son responsables, de un $56,5 \%$ a un $68,5 \%$, mientras que sus colegas investigadoras han visto reducidos paulatinamente los importes de sus proyectos, de un 43,4 a un 31,4 por cien. En el gráfico 2 se advierten claramente cuáles son las cuantías de tales contratos, por sexos, para cada uno de los intervalos elegidos, así como el sentido de la evolución operada en los mismos. 

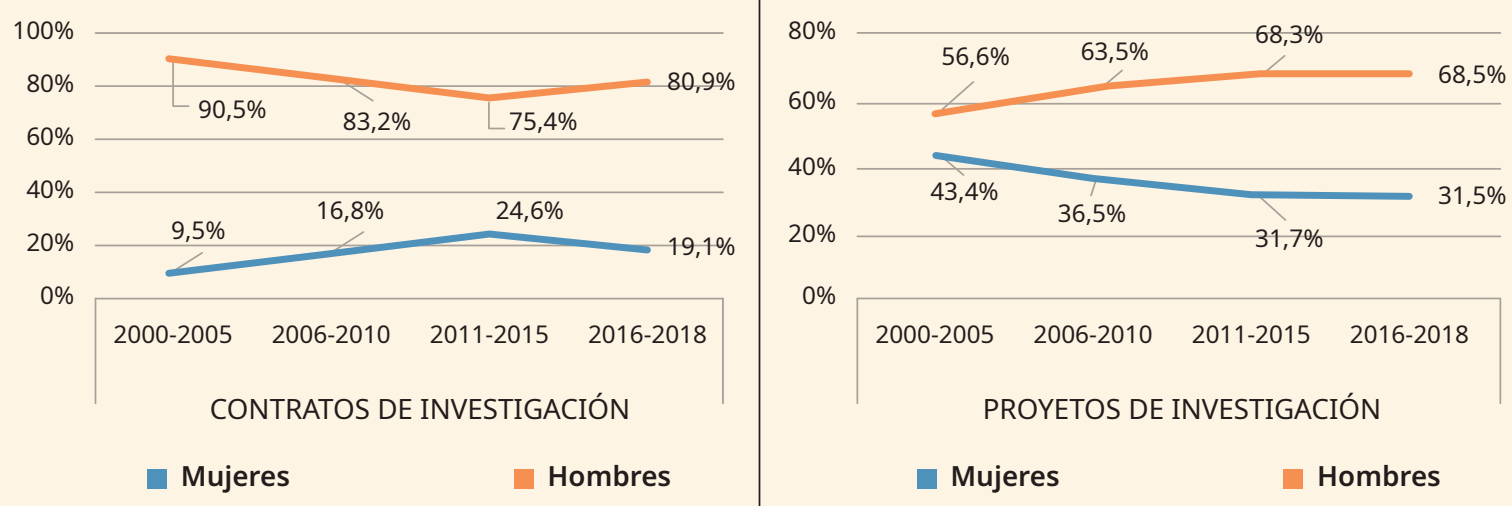

Fuente: Base de datos de investigación de la Universidad de Valencia (2019).

Cantidades mucho más elevadas de fondos de investigación y responsabilidad sobre un número mayor de proyectos, convenios y contratos de investigación son el balance de la desigualdad de género en el terreno de la investigación en ciencias de la salud en la Universidad de Valencia en el periodo analizado, mostrando un paisaje habitualmente común al del resto de las universidades españolas. Ello implica menor visibilidad de las mujeres científicas en ciencias médicas, menguando los referentes para que otras estudiantes mujeres sigan su camino, y ofrece la falsa conclusión de que el avance científico en disciplinas médicas es un logro masculino, retroalimentando y consolidando la desigualdad en un ámbito en cuyos estudios, en las Facultades españolas, existe desde hace algunas décadas un claro predominio femenino.

Si bien el liderazgo masculino y el predominio de los investigadores varones en el rol de investigador principal de los proyectos, contratos y convenios de investigación dotados con financiación son hechos indiscutibles, ello esconde una realidad en la que las académicas mujeres llevan el peso de los equipos, habida cuenta que predominan en su composición. En este sentido, un análisis complementario a los anteriores, de los datos del Registro de Estructuras de Investigación de la Universidad de Valencia, muestra que, en el área de Ciencias de la Salud, existen 70 grupos de investigación consolidados, y tan solo 30 (el 42,9\%) están dirigidos por académicas como investigadoras principales. En ellos participan 498 investigadores/as, de los que, 285, esto es, un 57,2 \% son mujeres. En definitiva, las académicas desarrollan importantes labores investigadoras integradas en grupos de investigación, la mayoría dirigidos por hombres, pero con un predominio cuantitativo de mujeres en su composición. Estas, sin embargo, poseen un techo de cristal a la hora de situarse en la posición de investigadoras principales, donde su número desciende, y todavía más a la hora de obtener fondos para sus proyectos, tal y como hemos visto, ya que en las iniciativas de investigación con financiación la preeminencia masculina es absoluta.

\section{Edad y categoría académica}

Por edades, y considerando tanto los proyectos como los contratos de investigación, se advierte un claro avance hacia situaciones prácticamente igualitarias en las generaciones más jóvenes e intermedias, frente al predominio absoluto de los hombres en las de mayor edad. Esta situación se observa particularmente en el número e importe de los proyectos de investigación liderados por mujeres y hombres de los diversos grupos de edad hasta 50 años, así como, para los contratos de investigación, en el colectivo de 41 a 50 años, en el que las mujeres han consolidado su carrera académica, liberándose de las responsabilidades familiares que conlleva la crianza de hijos/as en los primeros años. 
A partir de ahí, sin embargo, el predominio masculino es absoluto, tanto para proyectos como para contratos de investigación, ya en número de actividades investigadoras como en el importe de las mismas, en las cohortes que superan los 50 años, y particularmente en el personal investigador mayor de 60 años, donde la brecha se agranda estrepitosamente.

Varios factores explican estas tendencias. Ya se ha señalado que la financiación de los contratos suele obtenerse directamente, por encargo, mientras que la de los proyectos se obtiene de forma competitiva. En estos últimos, se aprecia una tendencia que apunta a posiciones más igualitarias entre hombres y mujeres, que se observa en las cohortes más jóvenes, en las que se puede responder a elementos como la valoración positiva de la paridad entre sexos en la evaluación de los proyectos a la incidencia de algunas políticas públicas de igualdad, como permisos y licencias idénticas para hombres y mujeres en materia de crianza de hijos e hijas, de implantación reciente en España, o a la importancia, cada vez mayor, que las propias académicas otorgan a sus carreras profesionales. Los datos experimentan, no obstante, variaciones por intervalos de edad, que se corresponden con diferentes fases de la carrera académica, ya que tras una etapa inicial igualitaria, en la que hombres y mujeres apuestan fuerte por encontrar su puesto en la universidad, desarrollando una labor investigadora importante, una vez conseguida la estabilidad, en muchos casos las mujeres suelen tener su primer hijo/a, hecho que puede ralentizar sus carreras profesionales en una segunda etapa de las mismas. Lo que es indiscutible es que, en las cohortes de mayor edad, los investigadores hombres han tenido y tienen una hegemonía absoluta, en importes y en número de proyectos y contratos de investigación. Para tales grupos de edad, en el momento de mayor eclosión de sus carreras, las mujeres no tuvieron tantas oportunidades como sus colegas varones. Quizá estas tendencias se alteren en unos años, en un sentido más igualitario, por lo que sería interesante continuar estudiándolas a lo largo del tiempo.

TABLA 1

PROYECTOS Y CONTRATOS DE INVESTIGACIÓN EN EL ÁREA DE CIENCIAS DE LA SALUD, POR SEXO, CATEGORÍA ACADÉMICA Y EDAD, 2000-2018

\begin{tabular}{|c|c|c|c|c|c|c|c|c|}
\hline & \multicolumn{3}{|c|}{$\begin{array}{c}\text { Número de } \\
\text { investigadores/as }\end{array}$} & \multicolumn{3}{|c|}{ Fondos de investigación } & \multicolumn{2}{|c|}{$\begin{array}{c}\text { Importe por } \\
\text { investigador/a }\end{array}$} \\
\hline & Total & $\begin{array}{c}\text { Hombres } \\
(\%)\end{array}$ & $\begin{array}{l}\text { Mujeres } \\
(\%)\end{array}$ & Total & $\begin{array}{c}\text { Hombres } \\
(\%)\end{array}$ & $\begin{array}{l}\text { Mujeres } \\
(\%)\end{array}$ & $\begin{array}{l}\text { Hombres } \\
(\%)\end{array}$ & $\begin{array}{l}\text { Mujeres } \\
(\%)\end{array}$ \\
\hline \multicolumn{9}{|c|}{ CATEGORÍA ACADÉMICA } \\
\hline \multicolumn{9}{|c|}{ Contratos de Investigación } \\
\hline $\begin{array}{c}\text { Ayudante Doctor/a } \\
\text { y otras figuras } \\
\text { contratado/a temporal }\end{array}$ & 32 & $68,8 \%$ & $31,3 \%$ & $723.654,6 €$ & $85,4 \%$ & $14,6 \%$ & $28.091,0 €$ & $10.565,3 €$ \\
\hline $\begin{array}{c}\text { Contratado Doctor/ } \\
\text { TU/CEU }\end{array}$ & 101 & $63,6 \%$ & $36,4 \%$ & $5.801 .767 €$ & $63,6 \%$ & $36,4 \%$ & $71.124,5 €$ & $35.693,5 €$ \\
\hline $\begin{array}{l}\text { Catedrático/a de } \\
\text { Universidad }\end{array}$ & 78 & $63,6 \%$ & $36,4 \%$ & $17.102 .703 €$ & $63,6 \%$ & $36,4 \%$ & $308.080,7 €$ & $84.610,0 €$ \\
\hline Profesor/a Emérito & 11 & $100,0 \%$ & --- & $935.441 €$ & $100,0 \%$ & --- & $85.040,1 €$ & --- \\
\hline \multicolumn{9}{|c|}{ Proyectos de Investigación } \\
\hline $\begin{array}{c}\text { Ayudante Doctor/a } \\
\text { y otras figuras } \\
\text { contratado/a temporal }\end{array}$ & 27 & $59,3 \%$ & $40,7 \%$ & $2.042 .369 €$ & $56,2 \%$ & $43,8 \%$ & $71.743 €$ & $81.317 €$ \\
\hline $\begin{array}{c}\text { Contratado Doctor/ } \\
\text { TU/ CEU }\end{array}$ & 71 & $52,1 \%$ & $47,9 \%$ & $6.642 .955 €$ & $61,5 \%$ & $38,5 \%$ & $110.353 €$ & $75.291 €$ \\
\hline $\begin{array}{l}\text { Catedrático/a de } \\
\text { Universidad }\end{array}$ & 79 & $60,8 \%$ & $39,2 \%$ & $30.236 .478 €$ & $65,9 \%$ & $34,1 \%$ & $415.361 €$ & $332.231 €$ \\
\hline Profesor/a Emérito & 5 & $100,0 \%$ & --- & $513.709 €$ & $100,0 \%$ & --- & $102.742 €$ & --- \\
\hline
\end{tabular}




\begin{tabular}{|c|c|c|c|c|c|c|c|c|}
\hline & \multicolumn{3}{|c|}{$\begin{array}{c}\text { Número de } \\
\text { investigadores/as }\end{array}$} & \multicolumn{3}{|c|}{ Fondos de investigación } & \multicolumn{2}{|c|}{$\begin{array}{c}\text { Importe por investiga- } \\
\text { dor/a }\end{array}$} \\
\hline & Total & $\begin{array}{l}\text { Hombres } \\
(\%)\end{array}$ & $\begin{array}{c}\text { Mujeres } \\
(\%)\end{array}$ & Total & $\begin{array}{l}\text { Hombres } \\
(\%)\end{array}$ & $\begin{array}{l}\text { Mujeres } \\
(\%)\end{array}$ & $\begin{array}{l}\text { Hombres } \\
(\%)\end{array}$ & $\begin{array}{l}\text { Mujeres } \\
(\%)\end{array}$ \\
\hline \multicolumn{9}{|c|}{ EDAD } \\
\hline \multicolumn{9}{|c|}{ Contratos de Investigación } \\
\hline De 30 a 40 & 18 & $50,0 \%$ & $50,0 \%$ & $128.542 €$ & $73,8 \%$ & $26,2 \%$ & $10.546 €$ & $3.737 €$ \\
\hline De 41 a 50 & 29 & $55,2 \%$ & $44,8 \%$ & $616.629 €$ & $37,6 \%$ & $62,4 \%$ & $14.490 €$ & $29.599 €$ \\
\hline De 51 a 60 & 91 & $58,2 \%$ & $41,8 \%$ & $9.616 .465 €$ & $73,6 \%$ & $26,4 \%$ & $133.594 €$ & $66.736 €$ \\
\hline Más de 60 & 92 & $77,2 \%$ & $22,8 \%$ & $14.411 .729 €$ & $91,9 \%$ & $8,1 \%$ & $186.557 €$ & $55.534 €$ \\
\hline \multicolumn{9}{|c|}{ Proyectos de Investigación } \\
\hline De 30 a 40 & 17 & $47,1 \%$ & $52,9 \%$ & $535.907 €$ & $50,6 \%$ & $49,4 \%$ & $33.879 €$ & $29.431 €$ \\
\hline De 41 a 50 & 32 & $46,9 \%$ & $53,1 \%$ & $3.174 .852 €$ & $46,3 \%$ & $53,7 \%$ & $97.946 €$ & $100.333 €$ \\
\hline De 51 a 60 & 84 & $53,6 \%$ & $46,4 \%$ & $21.183 .772 €$ & $57,3 \%$ & $42,7 \%$ & $269.891 €$ & $231.761 €$ \\
\hline Más de 60 & 45 & $73,3 \%$ & $26,7 \%$ & $16.629 .680 €$ & $83,5 \%$ & $16,5 \%$ & $420.768 €$ & $228.696 €$ \\
\hline
\end{tabular}

Fuente: Base de datos de investigación de la Universidad de Valencia (2019).

Por otro lado, uno de los filtros más interesantes a la hora de analizar los fondos de investigación universitarios en clave de género, sin duda lo constituye la categoría académica de los y las investigadores/ as. ${ }^{1}$ El estudio de los datos nos permite concluir que el rango académico sí que es un factor relevante, que incide particularmente en los proyectos de investigación, en los cuales se observa una pauta según la cual, en las categorías más elevadas (catedráticos y cated ráticas de universidad y profesores/as eméritos), existe un predominio masculino, tanto en importes como en número de proyectos, mientras que en otras figuras de rango inferior la situación que presentan hombres y mujeres es mucho más igualitaria. La consecución de fondos por sexo en cada una de las categorías académicas tiene una correlación directa con la proporción de mujeres en cada una de ellas, ya que aunque los datos de la tabla abarcan casi dos décadas, a lo largo de los cuales la cantidad de académicas mujeres en el área de ciencias de la salud en la Universidad de Valencia ha ido en aumento, lo cierto es que incluso en 2018, año final del análisis, tal y como ya se han señalado, las catedráticas de universidad mujeres solo representan el $26,2 \%$, mientras que en la categoría de profesorado emérito no hay ninguna mujer. Y en los otros dos grupos de profesorado utilizados para el análisis, el de Ayudante Doctor/a y otras figuras contratado/a de forma temporal, así como el de Contratado Doctor/ TU/ CEU, las académicas no alcanzan a ser más que 4 de cada 10. Esta desigual proporción por sexo en las diversas categorías de profesorado, y particularmente el hecho de que las mujeres solo representen una proporción muy discreta en las categorías más elevadas del colectivo de personal docente e investigador, que son quienes figuran como investigadores principales en la mayoría de proyectos que mayor volumen de financiación pública logran en las distintas convocatorias, explica el menor protagonismo femenino en la adjudicación de fondos de $\mathrm{I}+\mathrm{D}+\mathrm{i}$.

\footnotetext{
A efectos de analizar la distribución de fondos de investigación por categorías académicas, se han utilizado cuatro grupos. Los catedráticos y catedráticas de universidad son profesorado funcionario de la máxima categoría académica, del mismo modo que los profesores/a eméritos/as, que son profesorado jubilado que ha prestado servicios destacados a la universidad, y que puede seguir vinculado a la universidad por un máximo de seis años tras su jubilación. En este último grupo, que solo es accesible a un número limitado de personas, se integran habitualmente catedráticos/as de universidad con gran peso e influencia en la universidad, ya que deben ser designados como eméritos/as por el Consejo de Gobierno de la universidad en votación secreta. Un grupo intermedio en cuanto a categoría académica lo constituyen los y las docentes que son Contratados/as Doctores, Titulares de Universidad (TU) o Catedráticos de Escuela Universitaria (CEU), todos ellos del mismo nivel académico, al ser figuras estables, indefinidas, pero que no han alcanzado la máxima categoría, la de catedrático/a de universidad. Finalmente, la categoría académica más baja es la de Ayudante Doctor/a y otras figuras de contratación temporal, que tienen una vinculación máxima de 5 años con la universidad, por lo que, para continuar en la misma, deben promoverse a otras categorías de profesorado indefinidas.
} 
Ello no ocurre así en los contratos de investigación, en los que las diferencias por género, tanto en cuantías como en número de contratos, no siguen una pauta acorde con la categoría académica. Los investigadores hombres ostentan un predominio sobre sus colegas mujeres como investigadores principales de tales fondos para investigación médica, tanto en posiciones y rangos académicos intermedios, como en aquellos superiores. La razón de que los proyectos de investigación sí observen una relación directa con la categoría académica, a diferencia de los contratos, puede encontrarse en el hecho de que en aquellos los fondos se conceden de un modo competitivo, y los y las investigadores/as de mayor categoría suelen tener un mejor curriculum en punto a los elementos valorables en dichas convocatorias, al tiempo que pueden liderar mejores equipos de investigación a los que se adjudiquen tales fondos. En el caso de los contratos de transferencia de investigación, su consecución no deriva de un proceso de concurrencia competitiva, sino que su firma responde a otros elementos relacionales o de otra índole, no siendo tan relevante la categoría académica del investigador/a. La Tabla 1 muestra en detalle los resultados del estudio, considerando la categoría académica y la edad de los investigadores.

\section{Áreas clínicas}

Si introducimos como variable las especialidades clínicas a las que se destinan los fondos de investigación, lo primero que se constata es la existencia de un grupo de especialidades médicas que tan solo cuentan con investigadores varones. Ello ocurre en Cirugía, Dermatología, Oftalmología, Obstetricia y Ginecología, Otorrinolaringología, Radiología y Medicina Física, y Traumatología y Ortopedia. Además, en las áreas de Estomatología y de Medicina Legal y Forense, no existe ninguna investigadora que cuente con proyectos, tan solo están al frente de contratos de investigación. En el extremo contrario, encontramos una especialidad en la que solo existen académicas al frente de los fondos de investigación (Toxicología), mientras que en Pediatría los investigadores hombres solo cuentan con contratos de investigación, mas no con proyectos.

TABLA 2

PROYECTOS Y CONTRATOS DE INVESTIGACIÓN, POR SEXO Y ESPECIALIDADES CLÍNICAS, 2000-2018

\begin{tabular}{|c|c|c|c|c|c|c|}
\hline \multicolumn{7}{|c|}{ CONTRATOS DE INVESTIGACIÓN } \\
\hline \multirow{2}{*}{ Especialidad clínica } & \multicolumn{2}{|c|}{$\begin{array}{c}\text { Número de } \\
\text { investigadores/as }\end{array}$} & \multicolumn{2}{|c|}{ Fondos de investigación } & \multicolumn{2}{|c|}{$\begin{array}{l}\text { Importe por } \\
\text { investigador/a }\end{array}$} \\
\hline & Hombres & Mujeres & Hombres & Mujeres & Hombres & Mujeres \\
\hline \multirow{2}{*}{$\begin{array}{c}\text { Anatomía y Embriología } \\
\text { Humana }\end{array}$} & 6 & 4 & $735.674 €$ & $19.613 €$ & $122.612 €$ & $4.903 €$ \\
\hline & $60,0 \%$ & $40,0 \%$ & $97,4 \%$ & $2,6 \%$ & & \\
\hline \multirow{2}{*}{ Cirugía } & 8 & --- & $266.972 €$ & --- & $33.372 €$ & --- \\
\hline & $100,0 \%$ & --- & $100,0 \%$ & --- & & \\
\hline \multirow{2}{*}{ Dermatología } & 2 & -- & $21.600 €$ & --- & $10.800 €$ & --- \\
\hline & $100,0 \%$ & --- & $100,0 \%$ & --- & & \\
\hline \multirow{2}{*}{ Enfermería } & 10 & 4 & $125.603 €$ & $137.029 €$ & $12.560 €$ & $34.257 €$ \\
\hline & $71,4 \%$ & $28,6 \%$ & $97,4 \%$ & $2,6 \%$ & & \\
\hline \multirow{2}{*}{ Estomatología } & 9 & 5 & $1.974 .739 €$ & $91.279 €$ & $219.415 €$ & $18.256 €$ \\
\hline & $64,3 \%$ & $35,7 \%$ & $95,6 \%$ & $4,4 \%$ & & \\
\hline \multirow{2}{*}{$\begin{array}{c}\text { Farmacia y Tecnología } \\
\text { Farmacéutica }\end{array}$} & 7 & 8 & $270.697 €$ & $385.004 €$ & $38.671 €$ & $48.126 €$ \\
\hline & $46,7 \%$ & $53,3 \%$ & $41,3 \%$ & $58,7 \%$ & & \\
\hline \multirow{2}{*}{ Farmacología } & 9 & 10 & $7.957 .386 €$ & $683.279 €$ & $884.154 €$ & $68.328 €$ \\
\hline & $47,4 \%$ & $52,6 \%$ & $92,1 \%$ & $7,9 \%$ & & \\
\hline \multirow{2}{*}{ Fisiología } & 11 & 5 & $1.067 .733 €$ & $154.573 €$ & $97.067 €$ & $30.915 €$ \\
\hline & $68,8 \%$ & $31,3 \%$ & $87,4 \%$ & $12,6 \%$ & & \\
\hline
\end{tabular}


(continuación)

\begin{tabular}{|c|c|c|c|c|c|c|}
\hline \multirow{2}{*}{ Especialidad clínica } & \multicolumn{2}{|c|}{$\begin{array}{c}\text { Número de } \\
\text { investigadores/as }\end{array}$} & \multicolumn{2}{|c|}{ Fondos de investigación } & \multicolumn{2}{|c|}{$\begin{array}{l}\text { Importe por } \\
\text { investigador/a }\end{array}$} \\
\hline & Hombres & Mujeres & Hombres & Mujeres & Hombres & Mujeres \\
\hline \multirow{2}{*}{ Fisioterapia } & 5 & 6 & $567.886 €$ & $14.000 €$ & $113.577 €$ & $2.333 €$ \\
\hline & $45,5 \%$ & $54,5 \%$ & $97,6 \%$ & $2,4 \%$ & & \\
\hline \multirow{2}{*}{ Medicina } & 15 & 2 & $2.010 .360 €$ & $22.226 €$ & $134.024 €$ & $11.113 €$ \\
\hline & $88,2 \%$ & $11,8 \%$ & $98,9 \%$ & $1,1 \%$ & & \\
\hline \multirow{2}{*}{ Medicina Legal y Forense } & 4 & 1 & $201.829 €$ & $67.584 €$ & $50.457 €$ & $67.584 €$ \\
\hline & $80,0 \%$ & $20,0 \%$ & $74,9 \%$ & $25,1 \%$ & & \\
\hline \multirow{2}{*}{$\begin{array}{l}\text { Medicina Preventiva y } \\
\text { Salud Pública }\end{array}$} & 5 & 4 & $107.073 €$ & $490.130 €$ & $21.415 €$ & $122.532 €$ \\
\hline & $55,6 \%$ & $44,4 \%$ & $17,9 \%$ & $82,1 \%$ & & \\
\hline \multirow{2}{*}{ Microbiología } & 15 & 11 & $2.853 .613 €$ & $787.069 €$ & $190.241 €$ & $71.552 €$ \\
\hline & $57,7 \%$ & $42,3 \%$ & $78,4 \%$ & $21,6 \%$ & & \\
\hline \multirow{2}{*}{ Nutrición y Bromatología } & 5 & 9 & $683.587 €$ & $803.280 €$ & $136.717 €$ & $89.253 €$ \\
\hline & $35,7 \%$ & $64,3 \%$ & $46,0 \%$ & $54,0 \%$ & & \\
\hline \multirow{2}{*}{ Obstetricia y Ginecología } & 3 & --- & $250.171 €$ & --- & $83.390 €$ & --- \\
\hline & $100,0 \%$ & --- & $100,0 \%$ & --- & & \\
\hline \multirow{2}{*}{ Oftalmología } & 2 & --- & $2.728 €$ & --- & $1.364 €$ & --- \\
\hline & $100,0 \%$ & --- & $100,0 \%$ & --- & & \\
\hline \multirow{2}{*}{ Parasitología } & 4 & 3 & $92.058 €$ & $101.173 €$ & $23.014 €$ & $33.724 €$ \\
\hline & $57,1 \%$ & $42,9 \%$ & $47,6 \%$ & $52,4 \%$ & & \\
\hline \multirow{2}{*}{ Patología } & 8 & 4 & $570.403 €$ & $318.265 €$ & $71.300 €$ & $79.566 €$ \\
\hline & $66,7 \%$ & $33,3 \%$ & $64,2 \%$ & $35,8 \%$ & & \\
\hline \multirow{2}{*}{ Pediatría } & 1 & 2 & $18.000 €$ & $300 €$ & $18.000 €$ & $150 €$ \\
\hline & $33,3 \%$ & $66,7 \%$ & $64,2 \%$ & $35,8 \%$ & & \\
\hline \multirow{2}{*}{ Psiquiatría } & 8 & 1 & $479.567 €$ & $21.574 €$ & $59.946 €$ & $21.574 €$ \\
\hline & $88,9 \%$ & $11,1 \%$ & $64,2 \%$ & $35,8 \%$ & & \\
\hline \multirow{2}{*}{$\begin{array}{l}\text { Radiología y Medicina } \\
\text { Física }\end{array}$} & 1 & --- & $1.724 €$ & --- & $1.724 €$ & --- \\
\hline & $100,0 \%$ & --- & $100,0 \%$ & --- & & \\
\hline \multirow{2}{*}{ Traumatología y Ortopedia } & 3 & --- & $396.800 €$ & --- & $132.267 €$ & --- \\
\hline & $100,0 \%$ & --- & $100,0 \%$ & --- & & \\
\hline \multirow{2}{*}{ Toxicología } & --- & 2 & --- & $24.233 €$ & --- & $12.116 €$ \\
\hline & --- & $100,0 \%$ & --- & $100,0 \%$ & & \\
\hline \multicolumn{7}{|c|}{ PROYECTOS DE INVESTIGACIÓN } \\
\hline \multirow{2}{*}{$\begin{array}{c}\text { Anatomía y Embriología } \\
\text { Humana }\end{array}$} & 4 & 4 & $122.530 €$ & $55.000 €$ & $30.633 €$ & $13.750 €$ \\
\hline & $50,0 \%$ & $50,0 \%$ & $69,0 \%$ & $31,0 \%$ & & \\
\hline \multirow{2}{*}{ Cirugía } & 1 & --- & $56.265 €$ & --- & $56.265 €$ & --- \\
\hline & $100,0 \%$ & --- & $100,0 \%$ & --- & & \\
\hline Enformería & 4 & 2 & $83.175 €$ & $8.500 €$ & $20.794 €$ & $4.250 €$ \\
\hline & $66,7 \%$ & $33,3 \%$ & $90,7 \%$ & $9,3 \%$ & & \\
\hline Fstomatología & 5 & --- & $744.087 €$ & --- & $148.817 €$ & --- \\
\hline 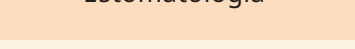 & $100,0 \%$ & --- & $100,0 \%$ & --- & & \\
\hline Farmacia y Tecnología & 5 & 5 & $288.413 €$ & $971.924 €$ & $57.683 €$ & $194.385 €$ \\
\hline Farmacéutica & $50,0 \%$ & $50,0 \%$ & $22,9 \%$ & $77,1 \%$ & & \\
\hline Farmarologí & 9 & 14 & $3.246 .779 €$ & $3.891 .544 €$ & $360.753 €$ & $277.967 €$ \\
\hline 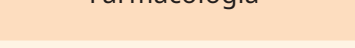 & $39,1 \%$ & $60,9 \%$ & $45,5 \%$ & $54,5 \%$ & & \\
\hline Fisioloaía & 14 & 9 & $7.311 .822 €$ & $351.029 €$ & $522.273 €$ & $39.003 €$ \\
\hline & $60,9 \%$ & $39,1 \%$ & $95,4 \%$ & $4,6 \%$ & & \\
\hline
\end{tabular}


(continuación)

\begin{tabular}{|c|c|c|c|c|c|c|}
\hline \multirow{2}{*}{ Especialidad clínica } & \multicolumn{2}{|c|}{$\begin{array}{c}\text { Número de } \\
\text { investigadores/as }\end{array}$} & \multicolumn{2}{|c|}{ Fondos de investigación } & \multicolumn{2}{|c|}{$\begin{array}{l}\text { Importe por } \\
\text { investigador/a }\end{array}$} \\
\hline & Hombres & Mujeres & Hombres & Mujeres & Hombres & Mujeres \\
\hline \multirow{2}{*}{ Fisioterapia } & 2 & 3 & $24.000 €$ & $21.935 €$ & $12.000 €$ & $7.312 €$ \\
\hline & $40,0 \%$ & $60,0 \%$ & $52,2 \%$ & $47,8 \%$ & & \\
\hline \multirow{2}{*}{ Medicina } & 14 & 3 & $2.551 .463 €$ & $508.419 €$ & $182.247 €$ & $169.473 €$ \\
\hline & $82,4 \%$ & $17,6 \%$ & $83,4 \%$ & $16,6 \%$ & & \\
\hline \multirow{2}{*}{ Medicina Legal y Forense } & 2 & --- & $133.962 €$ & --- & $66.981 €$ & --- \\
\hline & $100,0 \%$ & --- & $100,0 \%$ & --- & & \\
\hline \multirow{2}{*}{$\begin{array}{l}\text { Medicina Preventiva y } \\
\text { Salud Pública }\end{array}$} & 4 & 5 & $354.165 €$ & $816.065 €$ & $88.541 €$ & $163.213 €$ \\
\hline & $44,4 \%$ & $55,6 \%$ & $30,3 \%$ & $69,7 \%$ & & \\
\hline \multirow{2}{*}{ Microbiología } & 11 & 11 & $1.873 .941 €$ & $3.641 .652 €$ & $170.358 €$ & $331.059 €$ \\
\hline & $50,0 \%$ & $50,0 \%$ & $34,0 \%$ & $66,0 \%$ & & \\
\hline \multirow{2}{*}{ Nutrición y Bromatología } & 4 & 8 & $1.392 .725 €$ & $1.724 .496 €$ & $348.181 €$ & $215.562 €$ \\
\hline & $33,3 \%$ & $66,7 \%$ & $44,7 \%$ & $55,3 \%$ & & \\
\hline \multirow{2}{*}{ Obstetricia y Ginecología } & 2 & --- & $1.931 .680 €$ & --- & $965.840 €$ & --- \\
\hline & $100,0 \%$ & --- & $100,0 \%$ & --- & & \\
\hline \multirow{2}{*}{ Otorrinolaringología } & 1 & --- & $11.318 €$ & --- & $11.318 €$ & --- \\
\hline & $100,0 \%$ & --- & $100,0 \%$ & --- & & \\
\hline \multirow{2}{*}{ Parasitología } & 5 & 2 & $1.776 .399 €$ & $389.596 €$ & $355.280 €$ & $194.798 €$ \\
\hline & $71,4 \%$ & $28,6 \%$ & $82,0 \%$ & $18,0 \%$ & & \\
\hline \multirow{2}{*}{ Patología } & 6 & 7 & $2.176 .179 €$ & $1.053 .118 €$ & $362.696 €$ & $150.445 €$ \\
\hline & $46,2 \%$ & $53,8 \%$ & $67,4 \%$ & $32,6 \%$ & & \\
\hline \multirow{2}{*}{ Pediatría } & --- & 3 & --- & $205.845 €$ & --- & $68.615 €$ \\
\hline & --- & $100,0 \%$ & --- & $100,0 \%$ & & \\
\hline \multirow{2}{*}{ Psiquiatría } & 5 & 1 & $1.609 .006 €$ & $53.135 €$ & $321.801 €$ & $53.135 €$ \\
\hline & $83,3 \%$ & $16,7 \%$ & $96,8 \%$ & $3,2 \%$ & & \\
\hline \multirow{2}{*}{ Traumatología y Ortopedia } & 1 & --- & $68.285 €$ & --- & $68.285 €$ & --- \\
\hline & $100,0 \%$ & --- & $100,0 \%$ & --- & & \\
\hline \multirow{2}{*}{ Toxicología } & --- & 1 & --- & $61.297 €$ & $61.297 €$ & --- \\
\hline & --- & $100,0 \%$ & --- & $100,0 \%$ & & \\
\hline
\end{tabular}

Fuente: Base de datos de investigación de la Universidad de Valencia (2019).

Para el resto de las áreas médicas, en la inmensa mayoría de las mismas existe un claro predominio masculino tanto en proyectos como en contratos de investigación, que es todavía más acusado al considerar la diferencia en el importe de los fondos que reciben los académicos hombres y mujeres para investigar, que al cuantificar el número de investigadores por sexo para cada especialidad. Destacan, en ese sentido, Enfermería, Medicina y Fisiología, tanto en proyectos como en contratos; Anatomía y Embriología Humana, así como Farmacología y Fisioterapia en contratos de investigación; y Psiquiatría en proyectos: en casi todas estas áreas los investigadores varones concentran más del $90 \%$ de los fondos disponibles. Las investigadoras mujeres, por el contrario, disponen de más fondos para investigar en las especialidades de Farmacia y Tecnología Farmacéutica, Medicina Preventiva y Salud Pública y Nutrición y Bromatología, tanto procedentes de contratos como de proyectos de investigación; y en Microbiología, si solo consideramos estos últimos. En la Tabla 2 se advierten con nitidez todos los hechos y tendencias apuntados. 


\section{Retribuciones con cargo a contratos de transferencia de investigación}

En las universidades públicas españolas, las retribuciones ordinarias son necesariamente idénticas para hombres y mujeres en las diversas categorías. De ahí que una de las vías por las que pueden existir diferencias retributivas, y con ellas una brecha de género salarial, sea precisamente ésta, las retribuciones procedentes de los contratos de investigación.

El análisis de las cantidades percibidas por los investigadores con cargo a los contratos de $\mathrm{I}+\mathrm{D}+\mathrm{i}$, también arroja resultados profundamente desigualitarios. Si las científicas mujeres que han cobrado retribuciones complementarias con cargo a los contratos de $\mathrm{I}+\mathrm{D}+\mathrm{i}$ en el periodo objeto de estudio han sido 41, el número de académicos varones en la misma situación prácticamente triplica dicha cifra (son 117). Mucho más llamativa es la brecha entre los importes percibidos por unas et al., que ponen en evidencia una importante desigualdad entre ambos sexos (89.026,4 $€$ las científicas, 1.186.568,4 $€$ sus colegas hombres). O lo que es lo mismo, de cada $100 €, 93,02 €$ los han cobrado los varones, mientras que las investigadoras solo han percibido 6,98 €. El gráfico 3 muestra claramente la existencia y amplitud de esta brecha salarial.

Las situaciones de desigualdad de género conllevan que las científicas, en el terreno de las disciplinas médicas, consigan ser responsables de un número mucho menor de contratos de transferencia de investigación, y por esa vía perciban retribuciones sustancialmente menores que sus colegas varones

\section{GRÁFICO 3}

RETRIBUCIONES CON CARGO A CONTRATOS DE TRANSFERENCIA DE INVESTIGACIÓN EN CIENCIAS DE LA SALUD, POR SEXO, 2014-2018

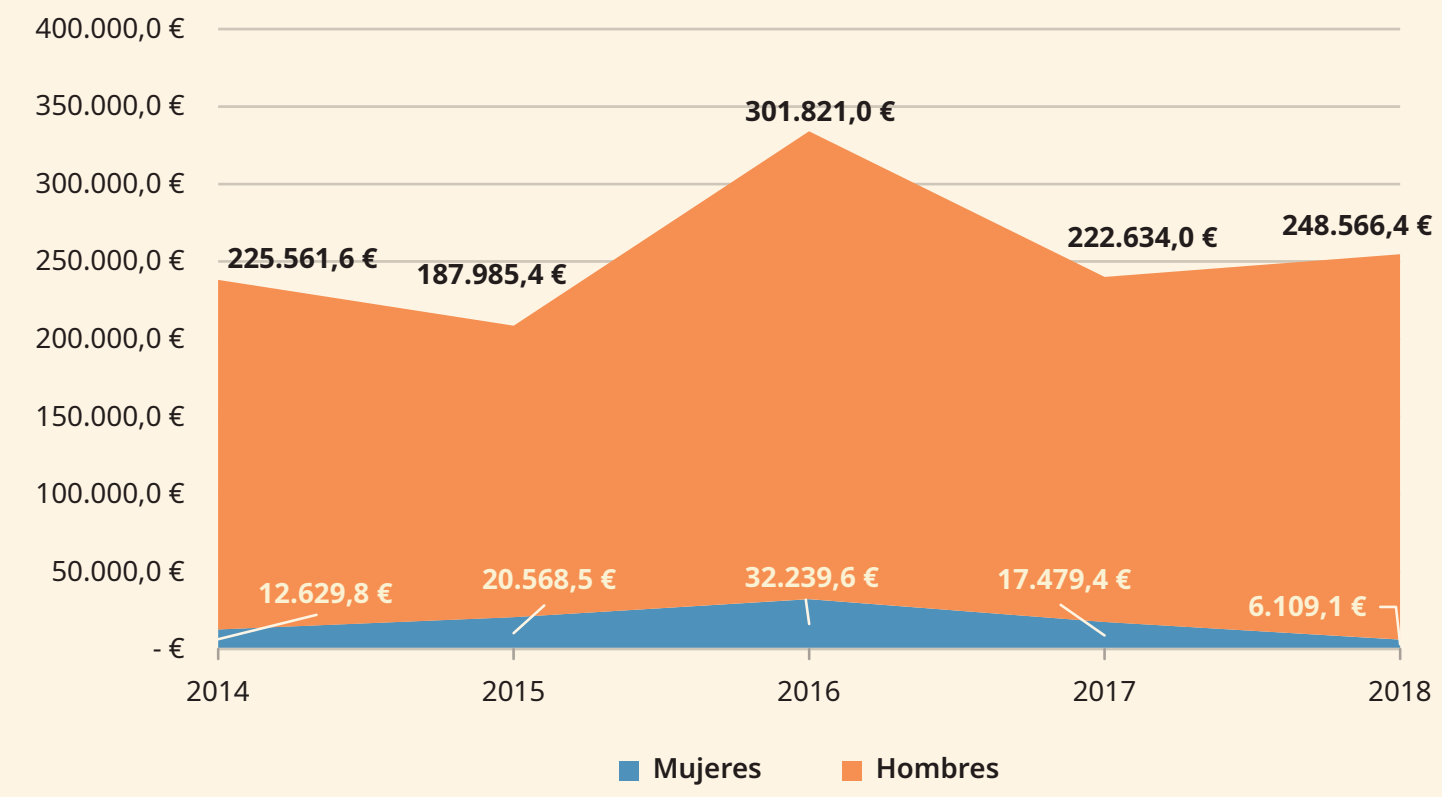

Fuente: Base de datos de nóminas de la Universidad de Valencia (2019).

\section{Conclusiones}

Los datos correspondientes a la Universidad de Valencia en el área de ciencias médicas, en una situación que probablemente coincida en lo esencial con otras universidades españolas, muestran que los profesores e investigadores hombres lideran de forma indiscutible los proyectos de investigación competitivos, así como los contratos de investigación a través de los cuales se transfieren los resultados del trabajo 
científico a la sociedad. Los académicos varones son responsables de un número abrumadoramente mayor de proyectos y de contratos y convenios de investigación, de importe también superior a aquellos liderados por sus colegas mujeres. Y ello, pese a que la participación de las académicas en los grupos de investigación de ciencias de la salud es mayor que las de sus colegas hombres, lo que revela que su trabajo sigue quedando en segundo plano, y que existe un techo de cristal cuyos efectos están plenamente vigentes.

Por edades, se advierte una situación ligeramente más igualitaria, especialmente por lo que respecta a los proyectos de investigación resultantes de convocatorias competitivas, en las generaciones más jóvenes e intermedias. También la categoría académica resulta relevante, ya que en el caso de los proyectos de investigación revela el importante predominio masculino, tanto en importes como en número de proyectos, más acusado en las categorías más elevadas (cátedras de universidad y profesorado emérito), que se suaviza en las categorías académicas inferiores, más tendientes a la paridad por sexos. Junto a ello, se constata una segregación horizontal que motiva que la investigación académica en un importante número de especialidades clínicas sea un territorio absolutamente masculino.

Finalmente, como consecuencia del mayor protagonismo de los investigadores varones, estos perciben con cargo a los contratos de investigación retribuciones adicionales a las ordinarias, comparativamente muy elevadas en relación a sus colegas académicas, generando así una importante brecha salarial de género.

El estudio ha confirmado, por tanto, la persistencia de un grado significativo de desigualdad de género en la investigación médica académica, con efectos perniciosos en múltiples facetas. Esta situación encuentra su explicación en una serie de argumentos, que sin duda continúan muy vigentes y poseen una importante fuerza, poniendo de manifiesto las barreras tanto personales como institucionales ya clásicas: las responsabilidades familiares; una imperfecta integración de las mujeres en la cultura académica, que resulta en menores destrezas a la hora de manejarse en un medio, la universidad, que tiene reglas propias de funcionamiento; escasa mentorización, desigual funcionamiento en equipos y grupos y pertenencia a lobbies académicos frente a los varones, mucho más diestros en el espacio profesional académico; en general, una planificación menos estratégica de sus carreras en el caso de las investigadoras, en comparación con sus colegas hombres; estereotipos y prejuicios sexistas más o menos conscientes, presentes en evaluaciones de investigación; o las representaciones vigentes en las ciencias médicas, que en determinadas facultades y especialidades son todavía masculinas, como lo es la idea de la excelencia científica.

Llaman la atención, sin embargo, los escasos progresos de la igualdad de género en la investigación en ciencias médicas en la universidad, considerando algunos factores que han cambiado claramente en la sociedad de España, y que debieran haber socavado algunas de las barreras personales e institucionales que impiden una mayor igualdad de género en la universidad española. El primero de ellos, el elevado número y la brillantez de las estudiantes mujeres de Medicina y de otras especialidades del área de la salud desde hace algunas décadas, cuyos efectos no se han visto reflejados en la proporción e importancia de las académicas en este ámbito. En segundo lugar, no resulta fácil medir exactamente el impacto de los factores familiares en el desarrollo de la carrera profesional de las académicas, dado que existen estudios con conclusiones diversas. Así, algunos trabajos empíricos minimizan su peso, considerando que no existen evidencias de que las mujeres con hijos e hijas registren una productividad científica y un rendimiento académico menor que aquellas que no los tienen, cuyas perspectivas de carrera académica siguen estando por detrás de las de sus colegas hombres (European Union, 2012). Otros datos y estudios, por el contrario, concluyen que las mujeres emplean una porción de su tiempo diario mucho mayor que los varones en la educación de sus descendientes y en la atención a la familia (a nivel europeo declaran hacerlo el 37,5 de las mujeres, frente al 24,7 \% de los hombres, cifras que en España son, respectivamente, del 39,8 y $27,7 \%$ ), así como a cocinar diariamente y a las labores del hogar $(78,7 \%$ de las mujeres, frente a solo el $33,7 \%$ de los varones, de media europea, indicadores que 
en España suponen, respectivamente, el 84,5 y el 41,9\%), dedicación temporal que indudablemente puede afectar a la esfera profesional (EIGE, 2019a). En todo caso, dicho impacto debería verse sustancialmente amortiguado por elementos como una -teórica- mayor implicación de los varones en la crianza de los hijos/as y en los temas domésticos, ${ }^{2}$ o por el hecho de que en España tenemos una de las tasas de fertilidad más bajas de la Unión Europea -1.31- (Eurostat, 2019). Además, las investigadoras académicas habitualmente pueden permitirse el disponer de ayuda externa para algunos cometidos de índole doméstica (Escolano, 2006). En el presente estudio, lamentablemente no se ha podido medir el impacto de las labores domésticas y la crianza de los hijos e hijas en la mayor o menor disponibilidad de fondos de investigación de las académicas involucradas, al no estar disponible dicho dato. Sería, por tanto, deseable, y sin duda muy interesante y útil para estudios diversos en clave de género e igualdad de oportunidades, el que el dato del número de hijos e hijas de los y las académicas, respetando en su utilización de forma estricta la normativa de protección de datos, pasara a formar parte de las bases de datos de las diversas instituciones educativas. Por último, en las universidades públicas españolas están vigentes desde hace ya algún tiempo numerosas e importantes normas, que establecen una igualdad entre los sexos en el terreno formal, que sin embargo quiebra flagrantemente en la práctica, tal y como certifican los datos ofrecidos. Y ello pese a la implantación de medidas de acción positiva presentes ya en las convocatorias de proyectos de investigación, competitivos, ya en los Planes de igualdad universitarios, que contienen de forma monográfica acciones dirigidas al objetivo de lograr la igualdad y combatir la discriminación en el campo de la investigación científica.

Todos los avances y transformaciones en este terreno no han conseguido cambiar el panorama de las universidades españolas en igualdad de género en ciencias de salud, revelando la extraordinaria resistencia de los obstáculos que actúan impidiendo una verdadera igualdad entre los académicos hombres y mujeres. Se impone explorar nuevas vías que permitan traspasar la superficie de lo formal y conseguir la igualdad real en la investigación médica universitaria, para que la academia sea un espacio de equidad capaz de aprovechar en beneficio de la sociedad el esfuerzo y el talento de las científicas médicas españolas.

\section{Fortalezas y limitaciones del presente estudio y dirección para futuras investigaciones}

Este estudio tiene fortalezas y limitaciones notables. Su limitación fundamental es que la investigación se ha llevado a cabo en una sola universidad española, aunque incluye datos de importantes Facultades y de numerosos departamentos universitarios en el campo de las Ciencias de la Salud. Esta característica impide la confirmación rigurosa de que sus conclusiones sean aplicables en general a las universidades españolas. Sin embargo, las características definitorias de la situación de las académicas en la institución analizada son muy similares a las del resto del país, en términos de número, rango académico, liderazgo e implicación en la cultura académica. Ello se puede comprobar, entre otras vías, analizando los datos sobre la composición del profesorado de dichas instituciones por sexo y categoría (en ambos casos, el acceso de las mujeres a la máxima categoría, la cátedra de universidad, en el ámbito de ciencias de la salud, dista mucho de presentar una situación igualiaria: si en el sistema universitario español en su conjunto las catedráticas de universidad son el 25,5\%, en la UV representan el 26,2\% -15,3\% en the Facultad de Medicina y

2 En este sentido, la legislación española ha avanzado de forma importante, mediante la concesión de permisos de paternidad y otras medidas tendentes a la conciliación y corresponsabilidad, aunque la implicación de los varones en las tareas domésticas y de crianza está todavía muy lejos de la que muestran las mujeres, y presenta un panorama menos igualitario que la media europea: 64, frente a 65,7 (EIGE, 2019a). 
Odontología, más del doble, 36,4 \%, en la Facultad de Farmacia-3³). De igual modo, los diagnósticos de las Unidades de Igualdad de las universidades españolas en materia de investigación y género, muestran un grado de desigualdad prácticamente coincidente (Red de Unidades de Igualdad de Género por la Excelencia Universitaria [RUIGEU], n.d.). Por otro lado, aunque el estudio se ciñe a la Universidad de Valencia, ésta es una institución académica muy relevante en el campo de las ciencias médicas.

Frente a esta limitación, resulta fundamental subrayar una fortaleza importante de este estudio, que es que no existe ningún trabajo en el campo de las ciencias de la salud que realice un análisis de género de los contratos universitarios de transferencia de los resultados de investigación. Tampoco se ha llevado a cabo ningún análisis que estudie las retribuciones adicionales que el personal docente e investigador percibe con cargo a los mismos, para determinar si existe una brecha salarial de género. Por todo ello, este estudio representa un paso inicial importante para abordar el desafío que supone acabar con las disparidades de género en la investigación académica médica. Es esencial realizar análisis rigurosos sobre este tema, con objeto de visibilizar la investigación científica de las investigadoras universitarias españolas en este campo esencial.

\section{Referencias}

Alloza Frutos, M., Anghel, B., Sánchez de Madariaga, I., Rica, S. de la, \& Dolado, J. J. (Coords.). (2012). White paper on the situation of women in science in Spain. Ministry of Science and Innovation. http://www. ciencia.gob.es/stfls/MICINN/Ministerio/FICHEROS/UMYC/WhitePaper_Interactive.pdf

Astegiano, J., Sebastián-González E., \& Castanho, C. T. (2019). Unravelling the gender productivity gap in science: A meta-analytical review. Royal Society Open Science, 6(6), Article e181566. http://doi.org/10.1098/rsos.181566

Bates, C., Gordon, L. K., Travis, E., Chatterjee, A., Chaudron, L., Fivush, B., Gulati, M., Jagsi, R., Sharma, P., Gillis, M., Ganetzky, R., Grover, A., Lautenberger, D., \& Moses, A. (2016). Striving for gender equity in academic medicine careers: A call to action. Academic Medicine, 91(8), 1050-1052. http:// doi.org/10.1097/ACM.0000000000001283

Besselaar, P. van de, \& Sandstrom, U. (2016). Gender differences in research performance and its impact on careers: A longitudinal case study. Scientometrics, 106, 43-62. https://doi.org/10.1007/s11192-015-1775-3

Bowen, C. J., Kersbergen, C. J., Tang, O., Cox, A., \& Beach, M. C. (2018). Medical school research ranking is associated with gender inequality in MSTP application rates. BMC Medical Education, 18(1), 187. https://doi.org/10.1186/s12909-018-1306-z

Buckley, L. M., Sanders, K., Shih, M., \& Hampton, C. L. (2000). Attitudes of clinical faculty about career progress, career success and recognition, and commitment to academic medicine: Results of a survey. Archives of Internal Medicine, 160(17), 2625-2629. https://doi.org/10.1001/archinte.160.17.2625

Butkus, R., Serchen, J., Moyer, D. V., Bornstein, S. S., \& Hingle, S. T. (2018). Achieving gender equity in physician compensation and career advancement: A position paper of the American College of Physicians. Annals of Internal Medicine, 168(10), 721-723. https://doi.org/10.7326/M17-3438

Carr, P. L., Raj, A., Kaplan, S. E., Terrin, N., Breeze, J. L., \& Freund, K. M. (2018). Gender differences in academic medicine: Retention, rank, and leadership comparisons from the national faculty survey. Academic Medicine, 93(11), 1694-1699. https://doi.org/10.1097/ACM.0000000000002146

Ceci, S. J., \& Williams, W. M. (2011). Understanding current causes of women's underrepresentation in science. Proceedings of the National Academy of Sciences, 108(8), 3157-3162. https://doi.org/10.1073/ pnas. 1014871108

3 Todos estos datos van refereridos al último año de nuestro estudio, esto es, al curso 2018-19, y se han extraído, respectivamente, del Recull de Dades Estadístiques de la Universidad de Valencia, y del Sistema Integrado de Información Universitaria estatal (SIIU) 
Connolly, S., \& Holdcroft, A. (2009). The pay gap for women in medicine and academic medicine: An analysis of the $W A M^{*}$ database. British Medical Association. https://www.medicalwomensfederation.org.uk/ images/Daonload_Pay_Gap_Report.pdf

Escolano, E. (2006). Entre la discriminación y el mérito: Las profesoras en las universidades valencianas. Publicaciones de la Universidad de Valencia.

European Commission. (2019). She figures 2018. https://ec.europa.eu/info/publications/she-figures-2018_en.

European Institute for Gender Equality. (2016). Promoting gender equality in academia and research institutions. https://eige.europa.eu/publications/promoting-gender-equality-academia-and-research-institutionsmain-findings.

European Institute for Gender Equality. (2019a). Gender Equality Index 2019. https://eige.europa.eu/genderequality-index/2019

European Institute for Gender Equality. (2019b). Tackling the gender pay gap: Not without a better work-life balance.Luxembourg: Publications Office of the European Union.https://eige.europa.eu/publications/ tackling-gender-pay-gap-not-without-better-work-life-balance

European Union. (2012). Meta-analysis of gender and science research. https://ec.europa.eu/research/swafs/pdf/ pub_gender_equality/meta-analysis-of-gender-and-science-research-synthesis-report.pdf

Eurostat (2019). Database Eurostat. https://ec.europa.eu/eurostat/data/browse-statistics-by-theme

Fox, M. F., Fonseca, C., \& Bao, J. (2011). Work and family conflict in academic science: Patterns and predictors among women and men in research universities. Social Studies of Science, 41(5), 715-735.

Lee, R. van de, \& Ellemers, N. (2015). Gender contributes to personal research funding success in the Netherlands. Proceedings of the National Academy of Sciences USA, 112, 12349-12353. https://doi. org/10.1073/pnas.1510159112

Leslie, S. J., Cimpian, A., Meyer, M., \& Freeland, E. (2015). Expectations of brilliance underlie gender distributions across academic disciplines. Science, 347(6219), 262-265. https://science.sciencemag.org/ content $/ 347 / 6219 / 262$

Mauleón, E., Hillán, L., Moreno, L., Gómez, I., \& Bordons, M. (2013). Assessing gender balance among journal authors and editorial board members. Scientometrics, 95(1), 87-114. https://doi.org/10.1007/ s11192-012-0824-4

Ministerio de Ciencia, Innovación y Universidades (2018a). Cientificas en cifras 2017. Estadisticas e indicadores de la (des)igualdad de género en la formación y profesión cientifica. http://www.ciencia.gob.es/stfls/ MICINN/Ministerio/FICHEROS/UMYC/Cientificas_cifras_2017.pdf

Ministerio de Ciencia, Innovación y Universidades (2018b). Sistema Integrado de Información Universitaria. https://www.ciencia.gob.es/portal/site

Monroe, A. K., Levine, R. B., Clark, J. M., Bickel, J., MacDonald, S. M., \& Resar, L. M. S. (2015). Through a gender lens: A view of gender and leadership positions in a department of medicine. Journal of Women's Health (Larchmt), 24(10), 837-842. http://doi.org/10.1089/jwh.2014.5054

Ovseiko, P. V., Greenhalgh, T., Adam, P., Grant, J., Hinrichs-Krapels, S., Graham, K. E., Valentine P. A., Sued O., Boukhris, O. F., Olaqi, N. M. Al, Rahbi, I. S. Al, Dowd, A-M., Bice, S., Heiden, T. L., Fischer, M. D., Dopson, S., Norton, R., Pollitt, A., Wooding, S., ... Buchan, A. M. (2016). A global call for action to include gender in research impact assessment. Health Research Policy and Systems, 14(1), 50. https:// doi.org/10.1186/s12961-016-0126-z

Pattani, R., Marquez, C., Dinyarian, C., Sharma, M., Bain, J., Moore, J. E., \& Straus, S. E. (2018). The perceived organizational impact of the gender gap across a Canadian department of medicine and proposed strategies to combat it: A qualitative study. BMC Medical Education, 16(1), 48. https://doi. org/10.1186/s12916-018-1032-8.

Paulus, J. K., Switkowski, K. M., Allison, G. M., Connors, M., Buchsbaum, R. J., Freund, K. M., \& Blazey-Martin, D. (2016). Where is the leak in the pipeline? Investigating gender differences in academic promotion at an academic medical centre. Perspectives on Medical Education, 5(2), 125-128. https:// doi.org/10.1007/s40037-016-0263-7 
Pingleton, S. K., Jones, V. M., Rosolowski, T. A., \& Zimmerman, M. K. (2016). Silent bias: Challenges, obstacles, and strategies for leadership development in academic medicine- Lessons from oral histories of women professors at the University of Kansas. Academic Medicine, 91(8), 1151-1157. https://doi. org/10.1097/ACM.0000000000001125

Plank-Bazinet, J. L., Bunker Whittington, K., Cassidy, S. K., Filart, R., Cornelison, T. L., Begg, L., \& Austin Clayton J. (2016). Programmatic efforts at the National Institutes of Health to promote and support the careers of women in biomedical science. Academic Medicine, 91(8), 1057-1064. https://doi. org/10.1097/ACM.0000000000001239

Raj, A., Carr, P. L., Kaplan, S. E., Terrin, N., Breeze, J. L., \& Freund, K. M. (2016). Longitudinal Analysis of Gender Differences in Academic Productivity among Medical Faculty across 24 Medical Schools in the United States. Academic Medicine, 91(8), 1074-1079. https://doi.org/10.1097/ ACM.0000000000001251

Red de Unidades de Igualdad de Género por la Excelencia Universitaria. (n.d.). La xarxa d'unitats d'igualtat de gènere per a l'excel.lència universitària. https://www.uv.es/ruigeu/ca/xarxa/es-red.html

Regulation (EU) no 1291/2013. (2013, 20 December). Of the European Parliament and of the Council of 11 December 2013 establishing Horizon 2020 - the Framework Programme for Research and Innovation (2014-2020) and repealing Decision No 1982/2006/EC. (Text with EEA relevance). Official Journal of the European Union. https://ec.europa.eu/research/participants/data/ref/h2020/legal_basis/fp/ h2020-eu-establact_en.pdf

Rochon, P. A., Davidoff, F., \& Levinson, W. (2016). Women in academic medicine leadership: Has anything changed in 25 years? Academic Medicine, 91(8), 1053-6. https://doi.org/10.1097/ ACM.0000000000001281

Shannon, G., Jansen, M., Williams, K., Cáceres, C., Motta, A., Odhiambo, A., Eleveld, A., \& Mannell, J. (2019). Gender equality in science, medicine, and global health: Where are we and why does it matter? Lancet, 393(10171), 560-569. https://doi.org/10.1016/S0140-6736(18)33135-0

Shen, H. (2013). Inequality quantified: Mind the gender gap. Despite improvements, female scientists continue to face discrimination, unequal pay and funding disparities. Nature, 495(7439), 22-24. https://doi. org/10.1038/495022a

Universidad de Valencia. (n.d.). Recull de Dades Estadistiques de la UV. https://www.uv.es/reculldedades

Waisbren, S. E., Bowles, H., Hasan, T., Zou, K. H., Emans, S. J., Goldberg, C., Gould, S., Levine, D., Lieberman, E., Loeken, M., Longtine, J., Nadelson, C., Patenaude, A. F., Quinn, D., Randolph, A. G., Solet, J. M., Ullrich, N., Walensky, R., Weitzman, P., \& Christou, H. (2008). Gender differences in research Grant applications and funding outcomes for medical school faculty. Journal of Women's Health, 17(2), 207-214. https://doi.org/10.1089/jwh.2007.0412

Wenneras, C., \& Wold, A. (1997). Nepotism and sexism in peer-review. Nature, 387(6631), 341-343. https:// doi.org/10.1038/387341a0

Westring, A., McDonald, J. M., Carr, P., \& Grisso, J. A. (2016). An integrated framework for gender equity in academic medicine. Academic Medicine, 91(8), 1041-1044. https://oi.org/10.1097/ ACM.0000000000001275

Yedidia, M. J., \& Bickel, J. (2001). Why aren't there more women leaders in academic medicine? The views of clinical department chairs. Academic Medicine, 76(5), 453-465. https://doi.org/10.1097/00001888200105000-00017

Zuckerman, H. (2001). The careers of men and women scientists: Gender differences in career attainment. In Wyer M. (Ed.), Women, science and technology: A reader in feminist science studies (pp. 69-78). Routledge. 


\section{Nota sobre autoría}

J. Eliseo Valle Aparicio (diseño del estudio, producción y análisis de datos, propuesta, redacción y revisión); Ángel Sanmartín Alonso (análisis de datos, propuesta, redacción y revisión); Raquel Valle Escolano (producción y análisis de datos, propuesta, redacción y revisión).

\section{Disponibilidad de datos}

Los datos de esta investigación están disponibles en: https://roderic.uv.es/handle/10550/74617

\section{Cómo citar este artículo}

Valle Aparicio, J. E., San Martín Alonso, A. \& Valle Escolano, R. (2021). Desigualdad de género en la investigación médica española: Un caso paradigmático. Cadernos de Pesquisa, 51, Artículo e07635. https://doi.org/10.1590/198053147635 